\title{
Network Analysis of Insomnia in Chinese Mental Health Professionals During the COVID-19 Pandemic: A Cross-Sectional Study
}

\author{
Wei Bai, ${ }^{1-3, *}$ Yanjie Zhao, ${ }^{1-3, *}$ \\ Fengrong An, ${ }^{4, *}$ Qinge Zhang, ${ }^{4, *}$ \\ Sha Sha, ${ }^{4} *$ Teris Cheung, ${ }^{5}$ \\ Calvin Pak-Wing Cheng, (1D ${ }^{6}$ \\ Chee $\mathrm{H} \mathrm{Ng}$, (D) ${ }^{7}$ Yu-Tao Xiang ${ }^{1-3}$ \\ 'Unit of Psychiatry, Department of Public Health \\ and Medicinal Administration, Institute of \\ Translational Medicine, Faculty of Health Sciences, \\ University of Macau, Macao SAR, People's Republic \\ of China; ${ }^{2}$ Centre for Cognitive and Brain Sciences, \\ University of Macau, Macao SAR, People's Republic \\ of China; ${ }^{3}$ Institute of Advanced Studies in \\ Humanities and Social Sciences, University of \\ Macau, Macao SAR, People's Republic of China; \\ ${ }^{4}$ The National Clinical Research Center for Mental \\ Disorders \& Beijing Key Laboratory of Mental \\ Disorders Beijing Anding Hospital \& the Advanced \\ Innovation Center for Human Brain Protection, \\ Capital Medical University, School of Mental \\ Health, Beijing, People's Republic of China; ${ }^{5}$ School \\ of Nursing, Hong Kong Polytechnic University, \\ Hong Kong SAR, People's Republic of China; \\ ${ }^{6}$ Department of Psychiatry, University of \\ Hong Kong, Hong Kong SAR, People's Republic of \\ China; ${ }^{7}$ Department of Psychiatry, The Melbourne \\ Clinic and St Vincent's Hospital, University of \\ Melbourne, Richmond, VIC, Australia \\ *These authors contributed equally to this work
}

Correspondence: Yu-Tao Xiang

Faculty of Health Sciences, University of Macau, I/F, Building EI2, Avenida da

Universidade, Taipa, Macao SAR, People's

Republic of China

Tel $+8538822-4223$

Fax $+8532288-2314$

Email xyutly@gmail.com

Chee $\mathrm{H} \mathrm{Ng}$

Department of Psychiatry, The

Melbourne Clinic and St Vincent's

Hospital, University of Melbourne,

Richmond, VIC, Australia

Email cng@unimelb.edu.au
Purpose: The coronavirus disease 2019 (COVID-19) pandemic is associated with increased risk of insomnia symptoms (insomnia hereafter) in health-care professionals. Network analysis is a novel approach in linking mechanisms at the symptom level. The aim of this study was to characterize the insomnia network structure in mental health professionals during the COVID-19 pandemic.

Patients and Methods: A total of 10,516 mental health professionals were recruited from psychiatric hospitals or psychiatric units of general hospitals nationwide between March 15 and March 20, 2020. Insomnia was assessed with the insomnia severity index (ISI). Centrality index (ie, strength) was used to identify symptoms central to the network. The stability of network was examined using a case-dropping bootstrap procedure. The network structures between different genders were also compared.

Results: The overall network model showed that the item ISI7 (interference with daytime functioning) was the most central symptom in mental health professionals with the highest strength. The network was robust in stability and accuracy tests. The item ISI4 (sleep dissatisfaction) was connected to the two main clusters of insomnia symptoms (ie, the cluster of nocturnal and daytime symptoms). No significant gender network difference was found. Conclusion: Interference with daytime functioning was the most central symptom, suggesting that it may be an important treatment outcome measure for insomnia. Appropriate treatments, such as stimulus control techniques, cognitive behavioral therapy and relaxation training, could be developed. Moreover, addressing sleep satisfaction in treatment could simultaneously ameliorate daytime and nocturnal symptoms.

Keywords: sleep, insomnia, SARS-CoV-2, COVID-19, physicians

\section{Introduction}

Insomnia, characterized by dissatisfaction with sleep quantity or quality, ${ }^{1}$ is a common sleep disorder. ${ }^{2}$ Several factors, such as mental disorders, ${ }^{3-5}$ emotional stress, ${ }^{6,7}$ and working at night or in shifts, ${ }^{8}$ are closely associated with insomnia. During the coronavirus disease 2019 (COVID-19) pandemic, health-care workers are confronted with the stress of high infection risk, heavy workload, long shift hours, and limited contact with their families, which can result in mental health issues, including stress, anxiety, depression, and insomnia. ${ }^{9-11}$ A recent metaanalysis reported that the pooled prevalence of insomnia in health-care workers during the COVID-19 pandemic was $34.32 \%,{ }^{12}$ which was higher than the general population. ${ }^{13}$ To reduce the risk of insomnia and develop effective measures to treat 
insomnia in this population, it is vital to understand the symptom patterns of insomnia.

Network theory is a promising novel approach to studying psychopathology and understanding linking mechanisms at a symptom level, ${ }^{14}$ in which the disorder is assumed to be constituted of co-occurring symptoms and that symptoms may trigger and accentuate each other. ${ }^{15}$ Unlike traditional approaches, which implicitly reflect the underlying latent factor ${ }^{16}$ or simply assume symptoms of disorders to be manifestations of an underlying disease, ${ }^{17,18}$ symptoms and their mutual interactions are seen as the disorder itself in the network approach. ${ }^{19}$ The network theory of psychopathology is flexible and dynamic and can explain the onset and maintenance of psychiatric symptoms. ${ }^{20}$ Therefore, the perspective of network approach offers the opportunities to illustrate the structure and functioning of important psychological phenomena ${ }^{16}$ such as the structure of insomnia symptoms. This theoretical framework can be statistically investigated via network analysis ${ }^{21}$ to visualize the structure of symptoms $^{22}$ and to reveal information or key symptoms related to clinical status rather than relying on the summation of total scores from scales. ${ }^{23}$

In network analysis, each symptom is viewed as one node and the interaction between two nodes is represented as an edge. The value of each edge stands for the statistical association calculated between two nodes after controlling for other nodes in the network structure. ${ }^{24}$ In the context of a strong edge in a network, the activation/deactivation of one symptom may predict the activation/deactivation of another symptom. ${ }^{25}$ In addition, the node position in the network represents its relative importance, so the more the node is centrally placed, the more interconnected the node is. Central symptoms are more prone to activate other symptoms; thus, they may play a pivotal role in causing the onset and/or maintenance of the syndrome. ${ }^{20}$ In theory, treatment targeting central symptoms are more effective than targeting peripheral symptoms. ${ }^{22}$ Consequently, network analysis is a highly valuable approach for depicting the psychological constructs and generating testable hypotheses for further studies. ${ }^{22,26}$

Network analysis has been used in diverse clinical conditions among specific populations such as anxiety and depression in adolescents ${ }^{27}$ and other psychiatric samples,${ }^{28}$ depressive symptoms in older people, ${ }^{20}$ hopelessness in a community sample, ${ }^{29}$ and post-traumatic stress disorder in earthquake survivors. ${ }^{19}$ To provide targeted interventions and strategies that facilitate prevention and alleviation of clinically significant distress, it is important to understand mechanisms that increase their risk for insomnia with sophisticated methods. A recent review $^{30}$ on the trends in insomnia research indicated research using network approach was warranted. To the best of our knowledge, only two studies ${ }^{31,32}$ have examined insomnia using network analysis. One network analysis explored the effect of personality traits on insomnia in the Netherlands, ${ }^{31}$ while the other network analysis explored the cognitive-behavioral therapy for insomnia (CBTI) induced effects on symptoms of insomnia and depression throughout the course of treatment. ${ }^{32}$ To date, no network analysis on insomnia in mental health professionals has been published.

Therefore, we conducted an exploratory study using a network analysis to examine the associations of insomnia symptoms (insomnia hereafter) and identify the most central (influential) symptoms that could serve as intervention targets in mental health professionals during the COVID19 pandemic.

\section{Materials and Methods Participants and Procedure}

This study was based on a secondary analysis of a national mental health survey of mental health professionals in China during the COVID-19 pandemic. ${ }^{33}$ To avoid the risk of contagion in population research, ${ }^{34,35}$ we recruited and assessed participants using snow sampling method from March 15, 2020 to March 20, 2020. The link to the invitation and assessment forms of this survey was distributed by the panel members of the Psychiatric Branch of the Chinese Nursing Association and the Chinese Society of Psychiatry to all public psychiatric hospitals in China via a quick response $(\mathrm{QR})$ code generated with the Questionnaire Star program embedded in WeChat. WeChat is a widely used communication application in China with around 1.2 billion users, including health-care professionals in clinical practice and continuing educational activities organized by the Chinese Nursing Association and the Chinese Society of Psychiatry. Therefore, nearly all mental health professionals working in psychiatric hospitals and psychiatric units of general hospitals in China were presumably WeChat users. The Psychiatry Branch, Chinese Nursing Association distributed the Quick Response Code (QR Code) linked to the assessment of this study to all psychiatric hospitals nationwide. Subsequently, mental health professionals working 
in these hospitals were invited to participate in the survey on an anonymous and voluntary basis.

Inclusion criteria included: (1) 18 years old and above; (2) mental health professionals (eg, doctors, nurses and nursing assistants) working in psychiatric hospitals or units in China during the COVID-19 pandemic; (3) able to understand Chinese and provide electronic written informed consent. No specific exclusion criteria were used in this study. The study protocol was approved by the Institutional Review Board (IRB) of Beijing Anding Hospital, China (2020 - Keyan (No. 10)) based on the local ethical regulations and the Declaration of Helsinki. ${ }^{36}$ All participants provided online written informed consent and the information collected were kept confidential.

\section{Measures}

Basic demographic information and clinical characteristics were collected, including age, gender, marital status, education level, living circumstances, type of hospital (tertiary or non-tertiary), and past experience combating Severe Acute Respiratory Syndrome (SARS) during the outbreak in 2003. Insomnia was measured by the 7-item Chinese version of the Insomnia Severity Index (ISI) questionnaire $^{37,38}$ which included 7 domains: 1) severity of sleep onset problem; 2) sleep maintenance problem; 3) early morning wakening problem; 4) sleep dissatisfaction; 5) interference of sleep difficulties with daytime functioning; 6) noticeability of sleep problems by others; and 7) distress caused by the sleep difficulties. Each item scored from 0 (no problem) to 4 (very severe problem); a higher score indicating more severe insomnia. The reliability and validity of the ISI has been well-documented in Chinese populations. ${ }^{38-40}$

\section{Statistical Analysis \\ Network Estimation}

The insomnia symptom network was estimated using the R program (Version 4.0.3). ${ }^{41}$ Following previous studies, ${ }^{25,29}$ item informativeness (ie, standard deviation (SD) of the item) was examined using the describe function in the R-package psych (Version 2.0.12), ${ }^{42}$ and item redundancy (ie, $<25 \%$ of statistically different correlations) was measured using the goldbricker function in the R-package networktools (Version 1.2.3). ${ }^{43}$ An item was regarded as having poor informativeness if its value is 2.5 times lower than the mean level of all items' informativeness in a scale, ${ }^{29}$ in this case, this item should be excluded from the network analysis.
In network parlance, each symptom is considered as node, and the correlations between two individual symptoms were considered edges. A thicker edge represents a stronger correlation, while the green color edge indicates a positive correlation, and red color indicates a negative correlation. ${ }^{44}$ The network structure of insomnia symptoms was estimated using the function estimateNetwork in the R-package bootnet (Version 1.4.3). ${ }^{24}$ As recommended previously, ${ }^{31}$ polychoric correlations were used to examine the correlations between ISI items, taking the Likert-scale type variables into account. To minimize spurious associations due to sampling error, the Extended Bayesian Information Criterion (EBIC) graphical least absolute shrinkage and selection operator (LASSO) model was adopted. $^{44}$ In this model, the LASSO algorithm is used to shrink all edges in the network and set small edges exactly to zero to make the network sparser and easier to interpret. ${ }^{45}$ The EBIC model selection approach has been widely used to select related tuning parameters ${ }^{24}(\gamma=0.5)$. To visualize the network, the R-package qgraph (Version 1.6.9) ${ }^{44}$ was used.

Centrality indices including strength, closeness, and betweenness were calculated to explore the importance of individual symptoms in the network using the R-package bootnet (Version 1.4.3). ${ }^{24}$ The default tuning parameter (alpha=1) was used. Following previous studies, ${ }^{24,29}$ in tandem with lower stability of betweenness and closeness, this study only focused on the strength index. The node strength refers to the sum of absolute weights of edges (expressed by correlation coefficients) connected to a certain node, indicating how strongly a node was directly connected to other nodes in the network. ${ }^{46}$ In addition, predictability was estimated using R-package mgm (Version $1.2-11),{ }^{47}$ indicating to what extent certain nodes were predicted by all its neighboring nodes.

\section{Network Stability and Accuracy}

The network stability and accuracy were assessed using the R-package bootnet (Version 1.4.3), ${ }^{24}$ which reflected the robustness of the results. Based on a case-dropping subset bootstrap procedure, the correlation stability coefficient (CS-C) was calculated for centrality indices. The value of $\mathrm{CS}-\mathrm{C}$ referred to the maximum proportion of dropped cases to maintain a correlation above 0.7 between the centrality indices of the original sample and those of subset samples with $95 \%$ of possibility. ${ }^{24}$ As recommended previously, ${ }^{24} \mathrm{CS}-\mathrm{C}$ value of above 0.25 is acceptable and preferably, above 0.5. A nonparametric 
bootstrap procedure was then used to assess the accuracy of edge weights. Edge accuracy was assessed by $95 \%$ confidence intervals (CIs), with a narrower CI indicating a more trustworthy network and a larger CI suggesting a poorer accuracy. ${ }^{24,29}$ In addition, bootstrapped tests were also conducted based on $95 \%$ CIs to evaluate the difference between two edges or between two nodes strength, with zero included in the CIs implying statistical differences. ${ }^{24}$

\section{Network Comparisons}

Considering the influence of gender on insomnia based on previous findings, ${ }^{48}$ three invariance measures (network structure invariance, global strength invariance, and edge invariance) of the network were examined using the Network Comparison Test (NCT) in the R-package NetworkComparisonTest 2.2.19 $1^{49}$ compare network measures between females and males. Network structure refers to the maximum difference of pairwise edges between female and male networks, global strength refers to the absolute sum of all edges of each network, and edge differences refer to the differences of individual edge weight between females and males networks. Holm-Bonferroni correction for multiple comparisons at the level of individual edge between gender was adopted. The three network comparison tests were conducted based on a similar permutation-based procedure. ${ }^{49}$ First, $50 \%$ of the participants in female and male dataset were randomly switched 1000 times to form the permutated datasets, which was considered as the reference distribution in the null hypothesis. Second, the three invariance measures (eg, network structure invariance, global strength invariance, and edge invariance) were calculated in the network structure estimate based on the original dataset (ie, observed statistics). Finally, the observed statistics were compared with the reference distribution created with the permutated procedure.

\section{Results}

\section{Descriptive Statistics}

Table 1 presents the demographic characteristics of participants. Of the 10,516 mental health professionals included in this network analysis, the mean age was 33.2 years ( $\mathrm{SD}=8.4), 1635$ (15.5\%) were males, 9635 (91.6\%) had college education or above, $7273(69.2 \%)$ were married, $8629(82.1 \%)$ lived with family, $948(9.0 \%)$ had past experiences combating SARS, and 6564 were working in tertiary hospitals. Information of the scale items are shown in the Supplementary Materials Table S1.
Table I Demographic Characteristics of Study Sample $(N=10,5 \mid 6)$

\begin{tabular}{|l|c|c|}
\hline Variables & Mean (SD) & N (\%) \\
\hline Age & $33.2(8.4)$ & - \\
Male gender & - & $1635(15.5)$ \\
College education and above & - & $9635(91.6)$ \\
Married status & & $7273(69.2)$ \\
Living with family & & $8629(82.1)$ \\
Past experience of SARS & - & $948(9.0)$ \\
Working in tertiary hospital & - & $6564(62.4)$ \\
\hline
\end{tabular}

Abbreviation: SD, standard deviation.

\section{Network Structure}

Item informativeness was calculated $\left(M_{S D}=0.8 \pm 0.1\right)$ and no item was found to be poorly informative. Additionally, analyses of item redundancy showed that no item was redundant with any other item in the ISI. Therefore, all the ISI items were included in the network analyses.

The network structure of insomnia is shown in Figure 1 and the corresponding correlation matric is presented in Table $\underline{\text { S2 }}$. All the connections showed positive associations between nodes. The edge ISI1 (severity of sleep onset) - ISI2 (sleep maintenance) has the strongest connection, followed by edge ISI2 (sleep maintenance) - ISI3 (early morning wakening problems), edge ISI5 (noticeability of sleep problems by others) - ISI6 (distress caused by the sleep difficulties), and the edge ISI6 (distress caused by the sleep difficulties) - ISI7 (interference with daytime functioning). Figure 1 shows two main clusters of insomnia, including the cluster of nocturnal symptoms (ISI1, ISI2, and ISI3), and the cluster of daytime symptoms (ISI5, ISI6, and ISI7), both of which were connected by the item ISI4 (sleep dissatisfaction).

In Figure 2, centrality indices of insomnia symptoms network show that ISI7 (interference with daytime functioning) was the most central symptom in mental health professionals with the highest strength. The item ISI3 (early morning wakening problems) was placed in the most peripheral area of the whole network with the lowest centrality indices. The predictability index shows that an average of $65.4 \%$ of variance could be potentially accounted by each node's neighboring nodes $\left(M_{\text {predictabikity }}=0.654 \pm 0.071\right)$. ISI7 (interference with daytime functioning, 73.6\%) had the highest predictability in the network shown in Table S1.

\section{Network Stability and Accuracy}

In terms of network stability shown in Figure S1, the CS-C of strength calculated by the case dropping bootstrap 


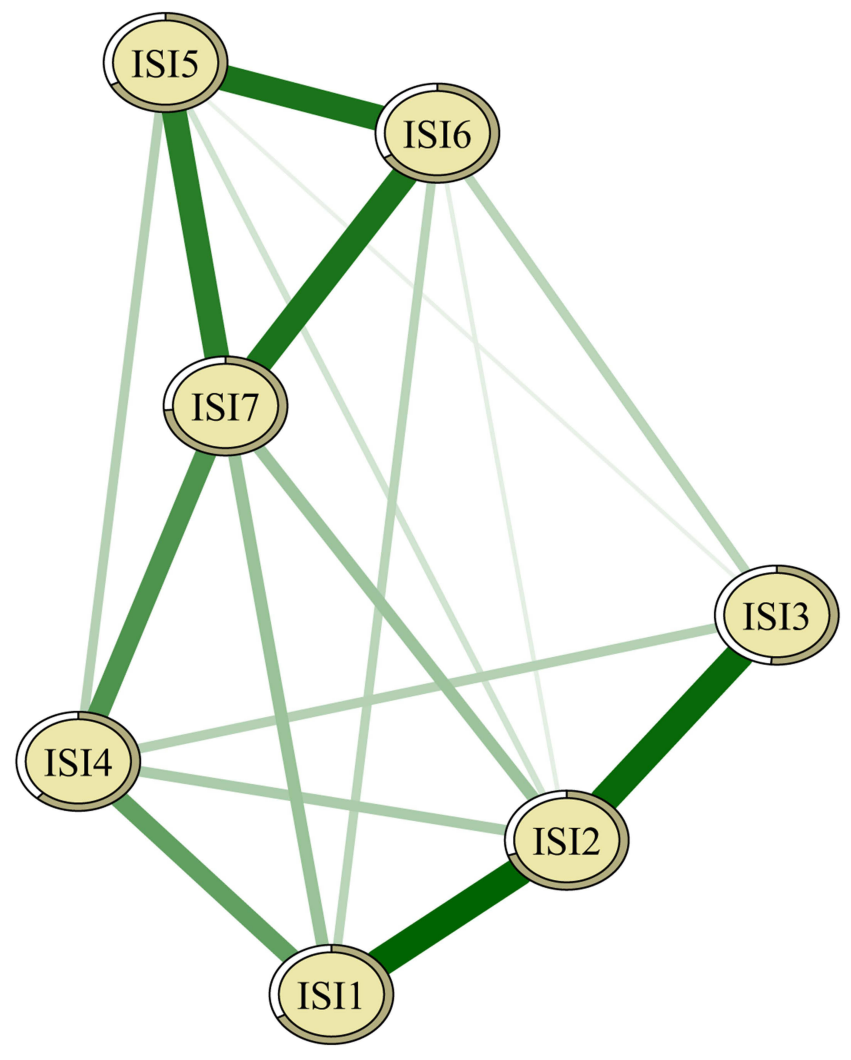

ISI1: Severity of sleep onset

ISI2: Sleep maintenance

ISI3: Early morning wakening problems

ISI4: Sleep dissatisfaction

ISI5: Noticeability of sleep problems by others

ISI6: Distress caused by the sleep difficulties

ISI7: Interference with daytime functioning

Figure I Estimated network model of insomnia symptoms in mental health professionals $(N=|0,5| 6)$.

procedure was 0.75 , which shows that the network remained stable, in that dropping $75 \%$ of sample would not change the primary results $(r=0.7)$. Regarding the accuracy of the present network, the results of bootstrap 95\% CI for edges show that 95\% CIs were narrow, indicating edges are trustworthy shown in Figure 3. The bootstrapped difference tests revealed that most comparisons among edge weights and node strength were statistically significant shown in Figure S2 and S3.

\section{Network Comparisons}

The NCT results did not find significant differences in the network global strength (females: 3.27 vs males: 3.30; $\mathrm{S}=0.03, p=0.48)$, network structure $(M=0.07, p=0.89)$, and individual edge weights (all $p$ values $>0.05$ after Holm-Bonferroni correction), indicated that network characteristics did not differ between genders. Plots are shown in supplementary files Figure S4-S6.

\section{Discussion}

This network analysis was the first exploratory study that characterized the network structure of insomnia in Chinese mental health professionals during the COVID-19 pandemic. Our results revealed that the symptoms were separated into two clusters connected by ISI4 (sleep dissatisfaction), and ISI7 (interference with daytime functioning) was the most central symptom in this sample.

In this study, we found that sleep-related problems (ie, ISI1: severity of sleep onset; ISI2: sleep maintenance; and ISI3: early morning wakening problems), also known as nocturnal symptoms, were rounded up in one cluster. On the other hand, the daytime symptoms (ISI5: noticeability of sleep problems by others; ISI6: distress caused by the sleep difficulties; ISI7: interference with daytime functioning) were organized into another cluster. These two clusters both contributed to the node ISI4 (sleep dissatisfaction). Our findings are consistent with the previous findings ${ }^{31}$ on the association of personality traits with different insomnia characteristics in the general population. These results suggest that the ISI total score may dilute the severity degree of nocturnal and daytime symptoms. ${ }^{31}$ Our study findings may have clinical significance, particularly for the treatment of insomnia. For instance, cognitive behavioral treatment of insomnia (CBT-I) is the most widely used non-drug treatment ${ }^{50}$ that encompasses techniques (ie, stimulus control 

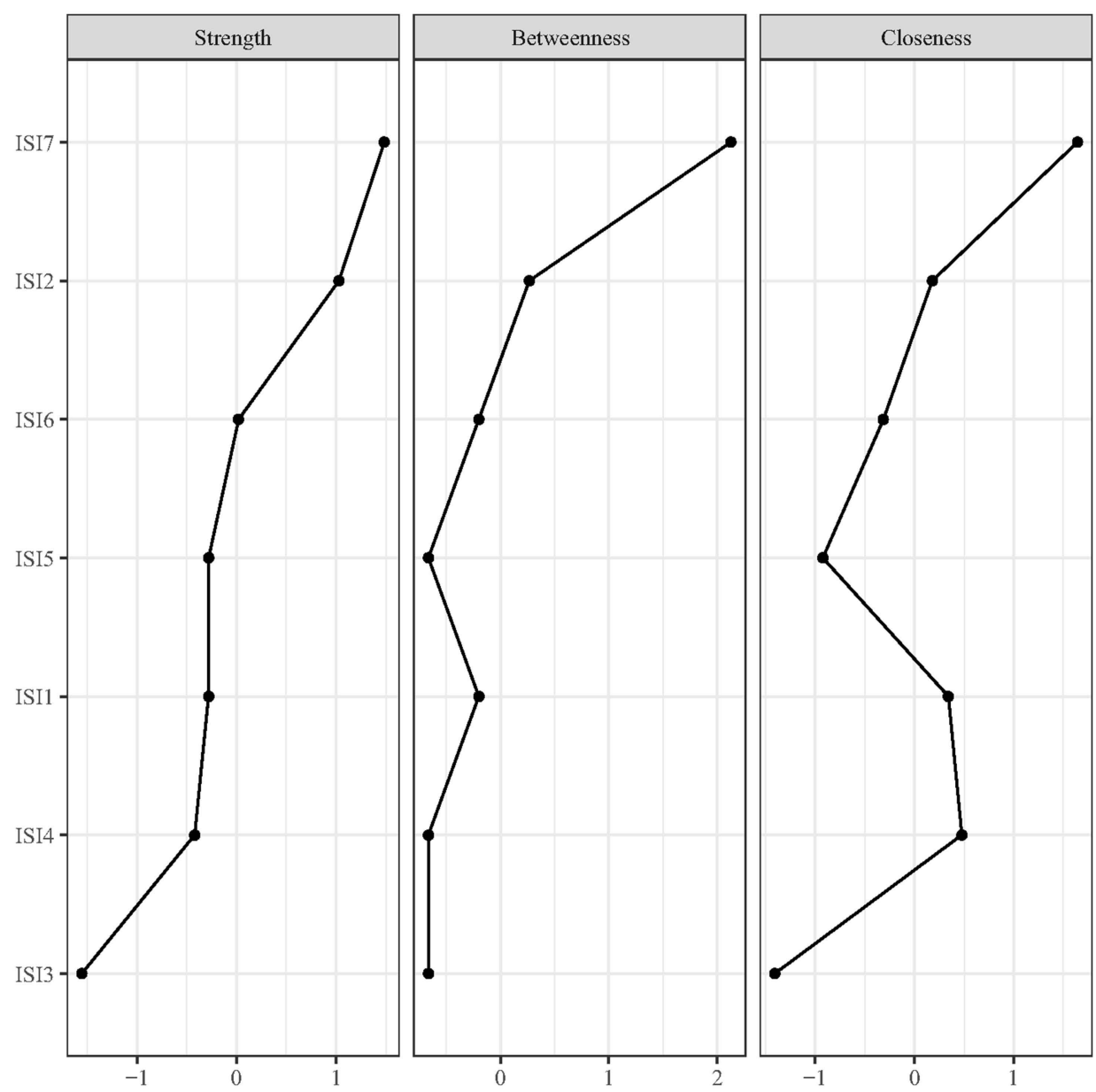

Figure 2 Centrality index of insomnia symptoms within the network.

Abbreviations: ISII, severity of sleep onset; ISI2, sleep maintenance; ISI3, early morning wakening problems; ISI4, sleep dissatisfaction; ISI5, noticeability of sleep problems by others; ISI6, distress caused by the sleep difficulties; ISI7, interference with daytime functioning.

techniques, cognitive therapy techniques, relaxation training) on managing expectations and beliefs in relation to nocturnal sleep improvement and coping strategies for daytime insomnia complaints. ${ }^{50,51}$ Hence, CBT-I could address both daytime and nocturnal symptoms. ${ }^{31}$ Based on our findings, if interventions are specifically targeted to address ISI4 (sleep dissatisfaction), daytime and nocturnal complaints may be ameliorated concurrently. ${ }^{31}$ Recent studies ${ }^{32,52}$ have also supported that research regarding the dynamic effects of interventions on specific symptoms of insomnia is further warranted.

The item ISI7 (interference with daytime functioning) was the most central (influential) symptom in this study. This survey was conducted immediately after the WHO's declaration of COVID-19 as a global pandemic ${ }^{53}$ when Chinese health-care workers were experiencing substantial stress due to the large number of COVID-19 cases and insufficient personal protective equipment. ${ }^{54,55}$ The item
ISI7 assessed daytime functioning interference (ie, ability to function at work/daily chores) caused by insomnia. Given the moral responsibility ${ }^{56}$ and strong sense of mission, health-care workers were likely concerned more about the negative consequences of insomnia problems, such as reduced work efficiency that may jeopardize patients' safety and medical service quality, ${ }^{57}$ than their experience of insomnia itself. Moreover, the edges ISI7-ISI5 (ie, interference with daytime functioning-noticeability of sleep problems by others) and ISI7-ISI6 (ie, interference with daytime functioning-distress caused by the sleep difficulties) showed strong connections in this network model, indicating that interventions targeting the two daytime complaints (ISI5 and ISI6) may improve the symptom of "interference with daytime functioning" (ISI7); hence, "interference with daytime functioning" (ISI7) could also be an important treatment outcome measure of insomnia. 


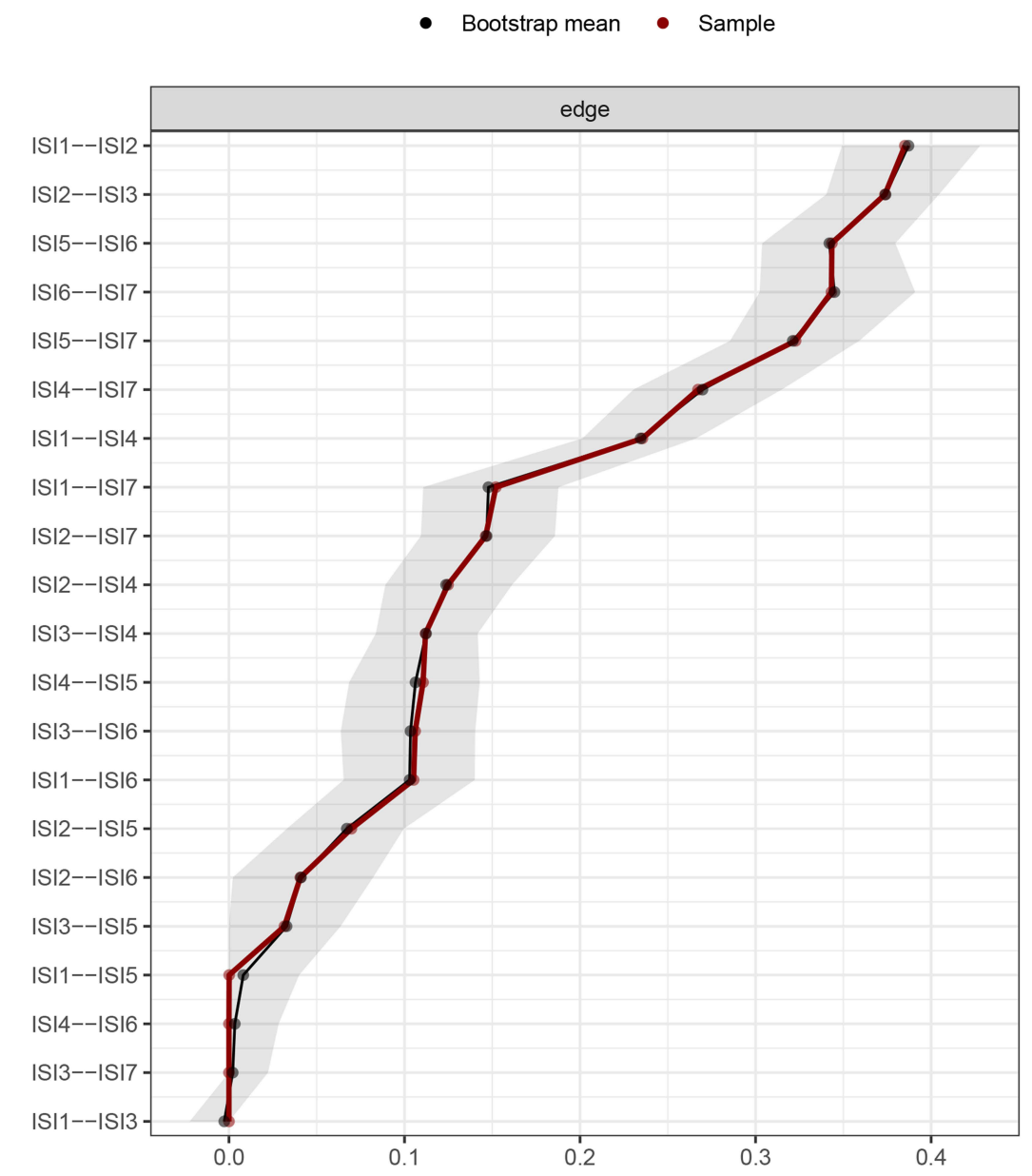

Figure 3 Bootstrapped confidence intervals of edge weights. The black dots indicate the values of each edge weight, ordered from the highest to the lowest value. The gray area represents the $95 \%$ confidence intervals of edge weights, estimated with the non-parametric bootstrap procedure.

Abbreviations: ISII, severity of sleep onset; ISI2, sleep maintenance; ISI3, early morning wakening problems; ISI4, sleep dissatisfaction; ISI5, noticeability of sleep problems by others; ISI6, distress caused by the sleep difficulties; ISI7, interference with daytime functioning.

The average of predictability is $65.4 \%$, indicating that most of the variance (ie, $65.4 \%$ ) of individual symptoms of insomnia could be accounted for by this network model. The remaining $(34.6 \%)$ variance could be attributed to other symptoms associated with insomnia, such as depressive and anxiety symptoms, fatigue, and perceived stress. ${ }^{48,58}$ Moreover, as recommended previously, ${ }^{29}$ the association of centrality index of strength and predictability with the item mean levels was examined, but no associations were found (node strength and item mean level: $\mathrm{r}_{\mathrm{s}}=-0.487, \mathrm{p}=0.268$; predictability and item mean level: $\left.r_{s}=-0.179, p=0.702\right)$. This suggests that network analysis could gain insight into the influence of individual symptoms on the model, which is not possible for traditional statistical approach that only used total scale scores; for example, the ISI7 (interference with daytime functioning) had the highest predictability and node strength in the network model, but its mean score was the lowest among all ISI items.
The strengths of this study included the large sample size and use of network analysis. Some limitations should be acknowledged. First, this is a cross-sectional study, hence the risk factors, co-morbidities and causality of the symptoms of insomnia could not be established. Second, data were collected based on participants' self-report, and the possibility of recall bias could not be excluded. Third, this study focused on mental health professionals, therefore, the findings could not be generalized to other populations, such as other health or general population. Third, an online survey with snowball sampling method was used to avoid the risk of COVID-19 infection, and therefore, the selection bias was inevitable with limited representativeness. Fourth, for logistical reasons, certain associated factors of insomnia, such as the different mental health disciplines, presence and severity of physical diseases, and the influence of insomnia on daily life, were not analyzed. 


\section{Conclusion}

The results of this network analysis may have treatment implications. As the symptom of interference with daytime functioning was the most central symptom in the insomnia model, it may be an important treatment outcome measure of insomnia in mental health professionals. Further, addressing sleep satisfaction in treatment could simultaneously improve daytime and nocturnal symptoms.

\section{Abbreviations}

$\mathrm{CI}$, confidence interval; $\mathrm{CS}-\mathrm{C}$, correlation stability coefficient; COVID-19, the coronavirus disease 2019; EBIC, Extended Bayesian Information Criterion; ISI, Insomnia Severity Index; LASSO, least absolute shrinkage and selection operator; NCT, Network Comparison Test; QR, quick response; SARS, Severe Acute Respiratory Syndrome; SD, standard deviation.

\section{Ethics Approval}

The study protocol was approved by the Institutional Review Board (IRB) of Beijing Anding Hospital, China (Approval No.: (2020) Keyan (No. 10)).

\section{Funding}

The study was supported by the National Science and Technology Major Project for investigational new drug (2018ZX09201-014), the Beijing Municipal Science \& Technology Commission (No. Z181100001518005), the University of Macau (MYRG2019-00066FHS), Faculty-allocated funds for PIs (FAF), and the Fundamental Research Funds for the Central Universities (2020YJ065).

\section{Disclosure}

The authors report no conflicts of interest in this work.

\section{References}

1. American Psychiatric Association. Diagnostic and Statistical Manual of Mental Disorders $\left(D S M-5^{\circledR}\right)$. American Psychiatric Pub; 2013.

2. Punnoose AR, Golub RM, Burke AE. Insomnia. JAMA. 2012;307 (24):2653. doi:10.1001/jama.2012.6219

3. Hertenstein E, Feige B, Gmeiner T, et al. Insomnia as a predictor of mental disorders: a systematic review and meta-analysis. Sleep Med Rev. 2019;43:96-105. doi:10.1016/j.smrv.2018.10.006

4. Pan X, Xiao Y, Ren D, et al. Prevalence of mental health problems and associated risk factors among military healthcare workers in specialized COVID-19 hospitals in Wuhan, China: a cross-sectional survey. Asia Pac Psychiatry. 2020:e12427. doi:10.1111/appy.12427

5. Um Min Allah N, Arshad S, Mahmood H, Abbas H. The psychological impact of coronavirus outbreak in Pakistan. Asia Pac Psychiatry. 2020;12(4):e12409. doi:10.1111/appy.12409
6. Basta M, Chrousos GP, Vela-Bueno A, Vgontzas AN. Chronic Insomnia and the Stress System. Sleep Med Clin. 2007;2 (2):279-291. doi:10.1016/j.jsmc.2007.04.002

7. Gu Y, Zhu Y, Xu F, Xi J, Xu G. Factors associated with mental health outcomes among patients with COVID-19 treated in the Fangcang shelter hospital in China. Asia Pac Psychiatry. 2021;13(2):e12443. doi:10.1111/appy.12443

8. Vallières A, Azaiez A, Moreau V, LeBlanc M, Morin CM. Insomnia in shift work. Sleep Med. 2014;15(12):1440-1448. doi:10.1016/j. sleep.2014.06.021

9. Chirico F, Ferrari G, Nucera G, Szarpak L, Crescenzo P, Ilesanmi O. Prevalence of anxiety, depression, burnout syndrome, and mental health disorders among healthcare workers during the COVID-19 pandemic: a rapid umbrella review of systematic reviews. J Health Soc Sci. 2021;6(2):209-220.

10. Chirico F, Nucera G, Magnavita N. Protecting the mental health of healthcare workers during the COVID-19 emergency. BJPsych Int. 2021;18(1). doi:10.1192/bji.2020.39

11. Magnavita N, Chirico F, Garbarino S, Bragazzi NL, Santacroce E, Zaffina S. SARS/MERS/SARS-CoV-2 Outbreaks and Burnout Syndrome among Healthcare Workers. An Umbrella Systematic Review. Int $J$ Environ Res Public Health. 2021;18(8):4361. doi:10.3390/ijerph18084361

12. Pappa S, Ntella V, Giannakas T, Giannakoulis VG, Papoutsi E, Katsaounou P. Prevalence of depression, anxiety, and insomnia among healthcare workers during the COVID-19 pandemic: a systematic review and meta-analysis. Brain Behav Immun. 2020;88:901-907. doi:10.1016/j.bbi.2020.05.026

13. Jahrami H, BaHammam AS, Bragazzi NL, Saif Z, Faris M, Vitiello MV. Sleep problems during the COVID-19 pandemic by population: a systematic review and meta-analysis. J Clin Sleep Med. 2021;17(2):299-313.

14. Shim EJ, Ha H, Park JE, et al. Gender-based examination of the association between individual symptoms of alcohol use disorder, major depressive disorder, and suicidal behaviors: a network analysis of data from the Korean Epidemiologic Catchment Area Study. $J$ Affect Disord. 2020;272:432-439. doi:10.1016/j. jad.2020.03.162

15. Borsboom D. A network theory of mental disorders. World Psychiatry. 2017;16(1):5-13. doi:10.1002/wps.20375

16. Schmittmann VD, Cramer AO, Waldorp LJ, Epskamp S, Kievit RA, Borsboom D. Deconstructing the construct: a network perspective on psychological phenomena. New Ideas Psychol. 2013;31(1):43-53. doi:10.1016/j.newideapsych.2011.02.007

17. Brown TA, Barlow DH. Dimensional versus categorical classification of mental disorders in the fifth edition of the Diagnostic and Statistical Manual of Mental Disorders and beyond: comment on the special section. $J$ Abnorm Psychol. 2005;114(4):551-556. doi:10.1037/0021-843X.114.4.551

18. Insel TR. The NIMH Research Domain Criteria (RDoC) Project: precision medicine for psychiatry. Am J Psychiatry. 2014;171 (4):395-397. doi:10.1176/appi.ajp.2014.14020138

19. McNally RJ, Robinaugh DJ, Wu GW, Wang L, Deserno MK, Borsboom D. Mental disorders as causal systems: a network approach to posttraumatic stress disorder. Clin Psychol Sci. 2015;3 (6):836-849. doi:10.1177/2167702614553230

20. Belvederi Murri M, Amore M, Respino M, Alexopoulos GS. The symptom network structure of depressive symptoms in late-life: results from a European population study. Mol Psychiatry. 2020;25 (7):1447-1456. doi:10.1038/s41380-018-0232-0

21. Epskamp S Network Psychometrics [dissertation]. University of Amsterdam; 2017. Available from: http://sachaepskamp.com/disserta tion/EpskampDissertation.pdf. Accessed October 16, 2021.

22. Borsboom D, Cramer AO. Network analysis: an integrative approach to the structure of psychopathology. Annu Rev Clin Psychol. 2013;9:91-121. doi:10.1146/annurev-clinpsy-050212-185608 
23. Tomba E, Bech P. Clinimetrics and clinical psychometrics: macroand micro-analysis. Psychother Psychosom. 2012;81(6):333-343. doi:10.1159/000341757

24. Epskamp S, Borsboom D, Fried EI. Estimating psychological networks and their accuracy: a tutorial paper. Behav Res Methods. 2018;50(1):195-212. doi:10.3758/s13428-017-0862-1

25. Mullarkey MC, Marchetti I, Beevers CG. Using Network Analysis to Identify Central Symptoms of Adolescent Depression. J Clin Child Adolescent Psychol. 2019;48(4):656-668. doi:10.1080/ 15374416.2018.1437735

26. Costantini G, Perugini M, Mõttus R. A framework for testing causality in personality research. Eur J Pers. 2018;32(3):254-268. doi:10.1002/per.2150

27. Dobson ET, Croarkin PE, Schroeder HK, et al. Bridging Anxiety and Depression: a Network Approach in Anxious Adolescents. J Affect Disord. 2021;280(Pt A):305-314. doi:10.1016/j.jad.2020.11.027

28. Beard C, Millner AJ, Forgeard MJ, et al. Network analysis of depression and anxiety symptom relationships in a psychiatric sample. Psychol Med. 2016;46(16):3359-3369. doi:10.1017/S0033291716002300

29. Marchetti I. Hopelessness: a Network Analysis. Cognit Ther Res. 2019;43(3):611-619. doi:10.1007/s10608-018-9981-y

30. Marques DR, Gomes AA, Clemente V, Dos Santos JM, Serra J, de Azevedo MHP. Trends in insomnia research for the next decade: a narrative review. Sleep Biol Rhythms. 2020;18(3):199-207. doi:10.1007/s41105-020-00269-7

31. Dekker K, Blanken TF, Van Someren EJ. Insomnia and Personality-A Network Approach. Brain Sci. 2017;7(3):28 doi:10.3390/brainsci7030028

32. Blanken TF, Van Der Zweerde T, Van Straten A, Van Someren EJW, Borsboom D, Lancee J. Introducing Network Intervention Analysis to Investigate Sequential, Symptom-Specific Treatment Effects: a Demonstration in Co-Occurring Insomnia and Depression. Psychother Psychosom. 2019;88(1):52-54. doi:10.1159/000495045

33. Xie XM, Zhao YJ, An FR, et al. Workplace violence and its association with quality of life among mental health professionals in China during the COVID-19 pandemic. J Psychiatr Res. 2021;135:289-293. doi:10.1016/j.jpsychires.2021.01.023

34. Kang L, Ma S, Chen M, et al. Impact on mental health and perceptions of psychological care among medical and nursing staff in Wuhan during the 2019 novel coronavirus disease outbreak: a cross-sectional study. Brain Behav Immun. 2020;87:11-17. doi:10.1016/j.bbi.2020.03.028

35. Zhang WR, Wang K, Yin L, et al. Mental Health and Psychosocial Problems of Medical Health Workers during the COVID-19 Epidemic in China. Psychother Psychosom. 2020;89(4):242-250. doi:10.1159/000507639

36. World Medical Association. World Medical Association Declaration of Helsinki: ethical Principles for Medical Research Involving Human Subjects. JAMA. 2013;310(20):2191-2194. doi:10.1001/ jama.2013.281053

37. Morin CM. Insomnia: Psychological Assessment and Management. Guilford press; 1993.

38. Yu DS. Insomnia Severity Index: psychometric properties with Chinese community-dwelling older people. J Adv Nurs. 2010;66 (10):2350-2359. doi:10.1111/j.1365-2648.2010.05394.x

39. Badiee Aval Baghyahi S, Torabi S, Gao Y, Kg C, Badiee Aval Baghsyahi HR. Reliability and validity of the Chinese translation of insomnia severity index (C-ISI) in Chinese patients with insomnia. Eur Psychiatry. 2011;26(S2):1556. doi:10.1016/S0924-9338(11)73260-6

40. Chung KF, Kan KK, Yeung WF. Assessing insomnia in adolescents: comparison of Insomnia Severity Index, Athens Insomnia Scale and Sleep Quality Index. Sleep Med. 2011;12(5):463-470. doi:10.1016/j. sleep.2010.09.019
41. R Core Team. R: a language and environment for statistical computing. R Foundation for Statistical Computing; 2020. Available from: https://www.R-project.org/. Accessed October 16, 2021.

42. Revelle W Procedures for Psychological, Psychometric, and Personality Research; 2020. Available from: https:/cran.r-project. org/web/packages/psych/index.html. Accessed October 16, 2021.

43. Payton J Tools for Identifying Important Nodes in Networks; 2020. Available from: https://cran.r-project.org/web/packages/networktools/ index.html. Accessed October 16, 2021.

44. Epskamp S, Cramer AOJ, Waldorp LJ, Schmittmann VD, Borsboom D. qgraph: network Visualizations of Relationships in Psychometric Data. J Stat Softw. 2012;48. doi:10.18637/jss.v048.i04

45. Tibshirani R. Regression shrinkage and selection via the lasso. J Royal Stat Soc. 1996;58(1):267-288.

46. Opsahl T, Agneessens F, Skvoretz J. Node centrality in weighted networks: generalizing degree and shortest paths. Soc Networks. 2010;32(3):245-251. doi:10.1016/j.socnet.2010.03.006

47. Haslbeck JMB, Waldorp LJ. mgm: estimating Time-Varying Mixed Graphical Models in High-Dimensional Data. J Stat Softw. 2020;93. doi:10.18637/jss.v093.i08

48. Zhan Y, Liu Y, Liu H, et al. Factors associated with insomnia among Chinese front-line nurses fighting against COVID-19 in Wuhan: a cross-sectional survey. J Nurs Manag. 2020;28(7):1525-1535. doi:10.1111/jonm.13094

49. van Borkulo CD, Boschloo L, Kossakowski JJ, et al. Comparing network structures on three aspects: a permutation test. Manuscript submitted for publication; 2017.

50. Buysse DJ. Insomnia. JAMA. 2013;309(7):706-716. doi:10.1001/ jama.2013.193

51. Morin CM, Bootzin RR, Buysse DJ, Edinger JD, Espie CA, Lichstein KL. Psychological and behavioral treatment of insomnia: update of the recent evidence (1998-2004). Sleep. 2006;29 (11):1398-1414. doi:10.1093/sleep/29.11.1398

52. Blanken TF, Jansson-Fröjmark M, Sunnhed R, Lancee J. Symptomspecific effects of cognitive therapy and behavior therapy for insomnia: a network intervention analysis. J Consult Clin Psychol. 2021;89:364-370. doi:10.1037/ccp0000625

53. Cucinotta D, Vanelli M. WHO Declares COVID-19 a Pandemic. Acta Bio-Medica. 2020;91(1):157-160.

54. Liu CY, Yang YZ, Zhang XM, et al. The prevalence and influencing factors in anxiety in medical workers fighting COVID-19 in China: a cross-sectional survey. Epidemiol Infect. 2020;148:e98. doi:10.1017/ S0950268820001107

55. Chen J, Liu X, Wang D, et al. Risk factors for depression and anxiety in healthcare workers deployed during the COVID-19 outbreak in China. Soc Psychiatry Psychiatr Epidemiol. 2021;56(1):47-55. doi:10.1007/s00127-020-01954-1

56. Spoorthy MS, Pratapa SK, Mahant S. Mental health problems faced by healthcare workers due to the COVID-19 pandemic-A review. Asian J Psychiatr. 2020;51:102119. doi:10.1016/j.ajp.2020.102119

57. Liu Q, Luo D, Haase JE, et al. The experiences of health-care providers during the COVID-19 crisis in China: a qualitative study. Lancet Global Health. 2020;8(6):e790-e798. doi:10.1016/S2214109X(20)30204-7

58. Lai J, Ma S, Wang Y, et al. Factors Associated With Mental Health Outcomes Among Health Care Workers Exposed to Coronavirus Disease 2019. JAMA Network Open. 2020;3(3):e203976. doi:10.1001/jamanetworkopen.2020.3976 


\section{Publish your work in this journal}

Nature and Science of Sleep is an international, peer-reviewed, open access journal covering all aspects of sleep science and sleep medicine, including the neurophysiology and functions of sleep, the genetics of sleep, sleep and society, biological rhythms, dreaming, sleep disorders and therapy, and strategies to optimize healthy sleep.

Submit your manuscript here: https://www.dovepress.com/nature-and-science-of-sleep-journ
The manuscript management system is completely online and includes a very quick and fair peer-review system, which is all easy to use. Visit http://www.dovepress.com/testimonials.php to read real quotes from published authors. 